\title{
TEM Assessments of the Restructuring Effects of an Emollient Cream on the stratum corneum
}

\author{
Richard Fitoussi $^{1}$, Katell Vie ${ }^{1}$, Éric Mathieu ${ }^{2}$, Éric Gooris ${ }^{1}$, Joseph Hemmerlé ${ }^{2}$ \\ ${ }^{1}$ Laboratoires Clarins, Pontoise, France; ${ }^{2}$ Institut National de la Santé et de la Recherche Médicale UMR 977, Université de Strasbourg, \\ Strasbourg, France. \\ E-mail: hemmerle@unistra.fr
}

Received October 13 ${ }^{\text {th }}$, 2011; revised November 21 ${ }^{\text {st }}$, 2011; accepted December $1^{\text {st }}, 2011$.

\begin{abstract}
The structure of the stratum corneum contributes to the barrier function of the epidermis. Skin barrier recovery is of utmost importance after epidermal tissue damage. The aim of this study was to describe, at the cellular level, the structural effects resulting from topical application of a hand-cream onto normal skin and to investigate the potential repair mechanisms induced by the emollient on altered tissue. Transmission electron microscopy (TEM) was used to compare the architectures of the horny layers from: 1) ex-vivo cultured human skin; 2) skin treated by topical application of a hand-cream emulsion; 3) explants exposed to sodium lauryl sulfate (SLS); 4) SLS-treated explants that underwent subsequent topical application of the emollient emulsion. These TEM assessments allowed identifying the structural changes occurring in the stratum corneum of skin explants exposed to SLS and/or treated with an emollient. Results strongly suggest that both, SLS-induced damage and emollient-driven repair process take place in the stratum corneum, at the cellular level. One can envisage that the observed restructuring effects after topical application of the skin-care product are likely to ameliorate or restore the barrier function of the stratum corneum. In this, the properties of the emollient go beyond the cosmetic feel.
\end{abstract}

Keywords: Emollient, Skin, stratum corneum, Corneocytes, TEM

\section{Introduction}

Skin barrier properties depend on several cellular and intercellular parameters. Epidermal cells are continuously renewed. Keratinocytes of the stratum granulosum become progressively cornified cells densely packed with keratin fibers. Epidermal desquamation is the final step in the differentiation program of the keratinocytes. The protein-rich nonviable corneocytes and the intercellular lipid domains protect the skin against chemical hazards. Irritant dermatitis can be induced by various agents. Chemicals such as detergents and/or organic solvents potentially perturb the stratum corneum in daily life. Sodium lauryl sulfate (Sodium dodecyl sulfate) is an anionic surfactant used in many cleaning and hygiene products. It has been shown to irritate the skin. Among others, its irritation potential is related to the surfactant concentration [1].

The aim of this study was to describe the structural changes resulting from topical application of a handcream onto normal skin and to draw the repair-mecha- nisms induced by the emollient on altered tissue. Topical applications have the advantage of targeting directly the area of interest that is the stratum corneum. Its structure contributes to the barrier function of the epidermis. Recent advances have been made in understanding stratum corneum lipid biophysics [2,3]. Special attention was paid to the organization of the intercellular lipids [4]. Although the intercellular lipids are increasingly studied with respect to their role in barrier function, little is actually known about the structural effect of the application of an emollient emulsion. Improving barrier function can be achieved by supplying lipid components to the stratum corneum. Cholesterol ceramides and fatty acids permeate into the nucleated cell layers of the skin and provide a delayed repair effect [5]. Mixtures of ceramides, cholesterol and fatty acids allow the barrier function to repair [6]. Couturier and Yvergnaux (2009) showed that the supply of missing ceramides by topical administration results in relipidization of the stratum corneum and leads to the regeneration of physiological processes [7]. On this matter, Fartasch (1997) pointed out that the lipid- 
rich intercellular structures of the stratum corneum might influence the desquamation process [8]. Lodén and Wessman (2001) studied the influence on skin barrier properties of a cream containing glycerine [9]. There results indicate an increased hydration of treated normal skin. According to Levi et al. (2009), the moisturizing glycerin reduces the drying stress in human stratum corneum [10].

Here we investigated the structural parameters of the stratum corneum in order to offer an insight into the repair processes going on in that skin-layer after emollient usage. The study was carried out by using ex vivo human skin explants, maintained in survival. The architecture of the stratum corneum in relation to skin damage by sodium lauryl sulfate (SLS) exposure and following repair possibilities by emollient applications were investigated by transmission electron microscopy (TEM).

\section{Materials and Methods}

\subsection{Ex Vivo Skin Culture}

Human skin specimens were obtained from abdomen reduction surgery of a 26-year-old Caucasian woman. Skin biopsies of $10 \mathrm{~mm}$ in diameter were taken from the specimens using biopsy punches. Skin explants from an abdominal plasty were placed in survival in classical cell culture conditions on stainless steel grids at the air-liquid interface, epidermal-side up. The grids were then placed in 6-well culture plates (Falcon) containing BEM medium (3.5 ml) (BioEC, Lonjumeaux, France) and cultured at $37^{\circ} \mathrm{C}$ in $5 \% \mathrm{CO}_{2}$ humidified air.

\subsection{Tissue Processing}

Standardized damage to the stratum corneum was caused with sodium lauryl sulfate (SLS) on skin explants. Explants were exposed for $24 \mathrm{~h}$ to the irritant using a 11 $\mathrm{mm}$ nonocclusive filter paper (patch) that was saturated with $0.1 \%$ aqueous solution of SLS (50 $\mu \mathrm{l})$. After $24 \mathrm{~h}$, patches were removed and each skin sample was gently rinsed with water and dried. An emollient emulsion was then applied $\left(2 \mathrm{mg} / \mathrm{cm}^{2}\right)$ daily, for 4 days, to some SLS exposed samples. Skin was treated topically with the emollient hand-cream "Crème jeunesse des mains" from Clarins, an oil-in-water emulsion containing $19 \%$ of fatty phase; in particular 5\% fatty alcohols, 3\% fatty alcohol esters and unsaponifiable fractions of shea butter and ceramides. One batch of explants was treated with the emollient cream without previous SLS application. As control, skin was topically treated with filter papers soaked in deionized water.

\subsection{Sample Process for TEM}

Punch biopsies were taken from the centers of the 3 types of specimens i.e. non-treated skin, tissue that underwent topical application of the hand-cream emulsion, SLS exposed skin and skin treated with the hand-cream after SLS exposure. Briefly, samples were fixed in a $2 \%$ glutaraldehyde solution buffered at $\mathrm{pH} 7.4$ with sodium cacodylate for electron microscopy (TEM), dehydrated in a series of increasing alcohol concentrations, post-fixed in $\mathrm{OsO}_{4}$ and finally embedded in epoxy resin. Ultra-thin sections were collected on carbon-coated formvar-covered cupper 100 mesh grids and stained with uranyl acetate and lead citrate. Transmission electron microscopy (TEM) was performed with a Philips EM 208 instrument (FEI Company, Eindhoven, The Netherlands) operating with an accelerating voltage of $70 \mathrm{kV}$.

\subsection{Image Analysis}

Digitalized micrographs were measured by an investigator who was blinded to the source of the samples. Measurements were performed by using a slide rule (Dafra type 941, Siemens, Germany) with a resolution of $10 \mu \mathrm{m}$. The stratum corneum was analyzed from micrographs taken at a magnification of 2000x; whereas measurements of the corneocytes come from micrographs taken at a magnification of $8000 \times$.

\subsection{Statistical Analysis}

Data analysis was performed using Student $t$-test by means of the computer software SigmaPlot 11.0 (Systat Software Inc., San Jose, CAL, USA).

\section{Results}

Four types of specimens were considered and compared in the present study. 1) ex-vivo cultured human skin from abdomen reduction surgery; 2) skin from the same plasty treated by topical application of a hand-cream emulsion; 3) cultured explants exposed to a $0.1 \%$ aqueous solution of sodium lauryl sulfate (SLS); 4) SLS treated skin explants that underwent subsequent topical application of the evaluated skin-care product. The architectures of the respective horny layers were assessed by transmission electron microscopy. Tissular and cellular thicknesses of the stratum corneum were measured and compared.

The bar graph of Figure 1 depicts the mean thickness of the stratum corneum vs skin treatment. It shows that the topical application of the emollient cream results in a thickening of the stratum corneum, both when applied directly or after SLS treatment on the skin explants. Figure 1 also indicates that the SLS exposure induces a thinning of the horny layer. The statistical differences between the various treatments (emollient application, SLS exposure and emollient application after SLS exposure) are highly significant ( $\mathrm{p} \leq 0.001)$. The architecture of the stratum corneum from non-treated skin is described 


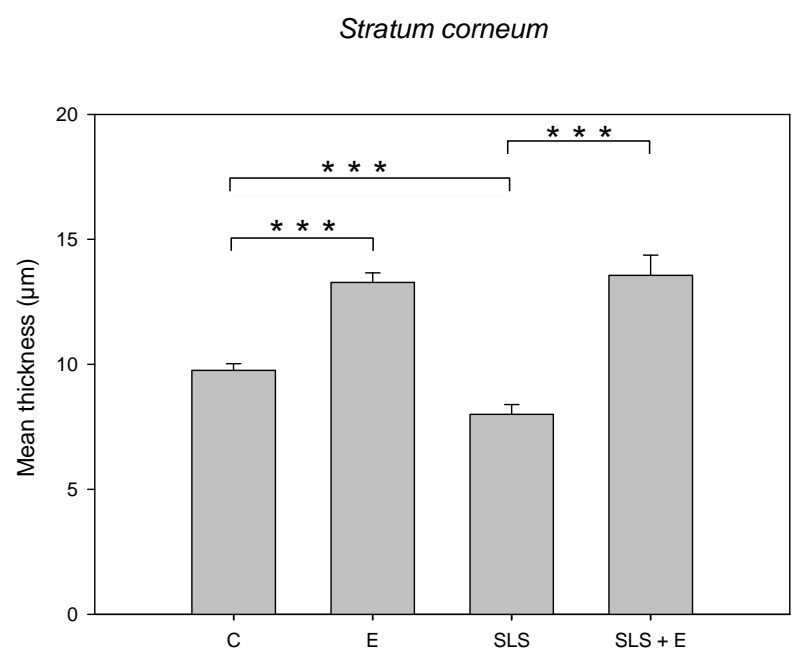

Figure 1. Bar graph depicting the mean thickness of the stratum corneum vs skin treatment. Control specimen (C). $n$ $=30$ measurements; emollient cream application $(\mathrm{E}), \boldsymbol{n}=\mathbf{3 0}$ measurements; SLS treatment (SLS), $n=30$ measurements; emollient application after SLS exposure (SLS + E), $n=30$ measurements. Error bars represent the SEM.

by Figure 2(a). It exhibits two distinct domains: an innermost electron-dense layer of corneocytes and an outermost more electron-lucent layer of cornified cells. The focal detachment of superficial corneocytes illustrates the physiological desquamation process. Figure 2(b) focuses on the electron-dense layer. Visual examination of the micrograph shows tightly packed keratin fibrils within the cornifed cells. Corneocytes are held together by corneodesmosomes that are clearly identifiable. Focal dilatations separate the intercellular ties. Corneodesmosomes are absent above the fifth corneocyte layer. Only corneodesmosome remnants are found between the superficial corneocytes of the electron-lucent layer (Figure 2(c)). It appears that the electron-lucent aspect of this outermost layer comes from the weak stain of the scattered intracellular keratin matrix. If one considers the thickness of this electron-lucent horny layer (Figure 3), it seems evident that the SLS solution acts mainly at this level. Measurements show that the weakly-bound electron-lucent cornified cells almost disappear after SLS treatment. The bar graph of Figure 3 points out that the topical application of the emollient emulsion also acts at this level by thickening the outermost layer of corneocytes, both when applied directly or after SLS exposure on the skin explants. The statistical differences between the various treatments (emollient application, SLS exposure and emollient application after SLS exposure) are highly significant ( $\mathrm{p} \leq 0.001)$. SLS damage induces drastic desquamation (Figure 4(a)). Almost all corneocytes from the outermost electron-lucent layer are shed from the skin surface. It results in turn in reduced thickness of

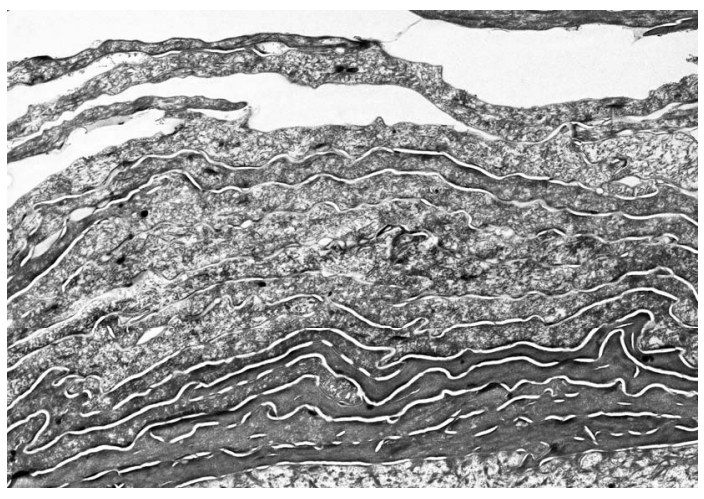

(a)

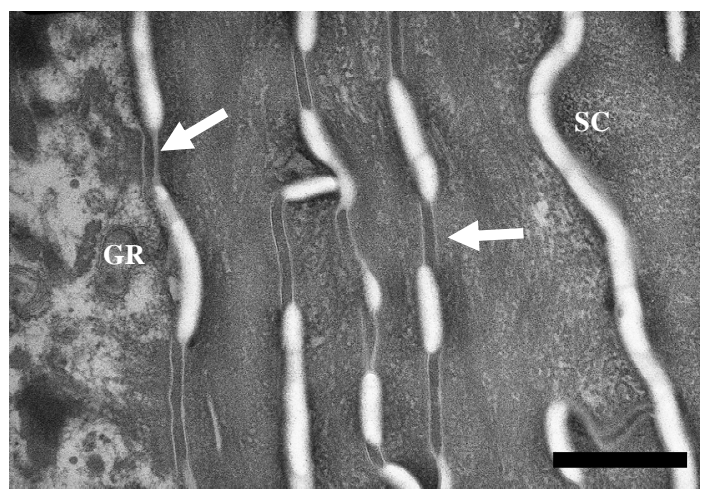

(b)

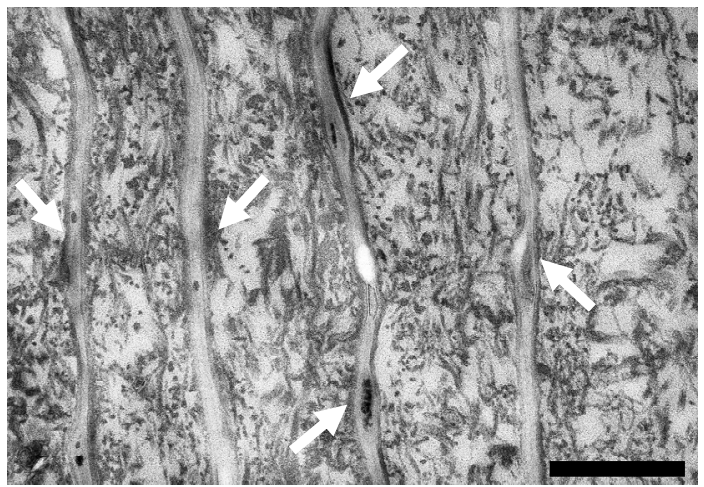

(c)

Figure 2. (a) TEM micrograph describing the architecture of the stratum corneum from non-treated skin. One can note electron-dense corneocytes at the top of the stratum granulosum and more electron-lucent corneocytes in the outermost horny layer. Scale bar represents 2 um; (b) Electron micrograph showing an electron-dense layer of corneocytes from the stratum corneum (SC) at the top of the keratinocytes from the stratum granulosum (GR). Corneodesmosomes are clearly identifiable (arrows). Nontreated skin explant. Scale bar represents $0.5 \mu \mathrm{m}$; (c) Electron micrograph showing the electron-lucent corneocytes of the most superficial layer of the stratum corneum. Intracellular keratin matrix appears disrupted. Only remnants of corneodesmosomes (arrows) are noticed between the parallel organized cornified cells. Non-treated skin. Scale bar represents $0.5 \mu \mathrm{m}$. 
Electron-lucent layer of stratum corneum

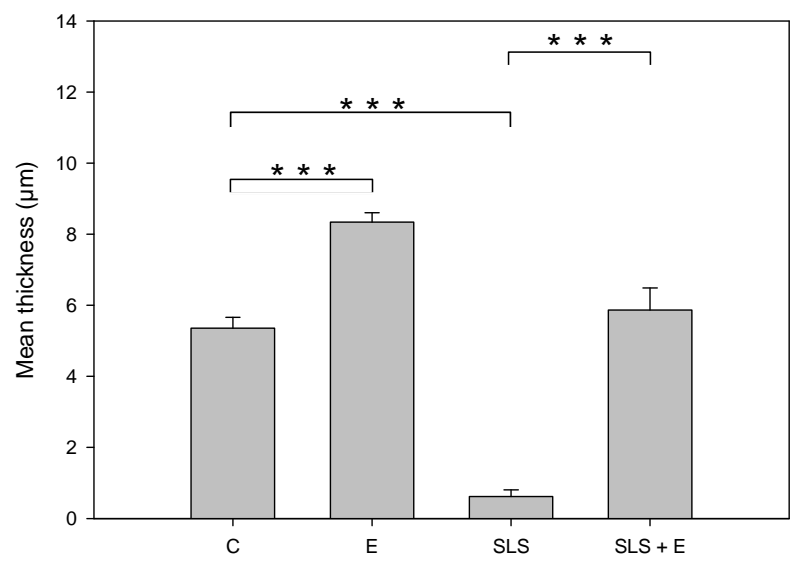

Figure 3. Bar graph depicting the mean thickness of the outermost electron-lucent layer of the stratum corneum vs skin treatment. Control specimen (C). $n=30$ measurements; emollient cream application ( $E), n=30$ measurements; SLS treatment (SLS), $n=30$ measurements; emollient application after SLS exposure (SLS + E), $n=30$ measurements. Error bars represent the SEM.

the horny layer of the epidermis. The accelerated shedding of cornified cells involves even electron-dense corneocytes from the innermost stratum corneum (Figure 4(b)). Scales are made of individual or groups of horny cells. Cornified envelopes of desquamated cells form spicules.

According to Figure 5(a) the architecture of the Stratum corneum appears unchanged after topical application of the hand-cream. Nevertheless, Figure 1 emphasizes that the mean thickness of the stratum corneum increases from $9.76 \pm 0.27 \mu \mathrm{m}$ for untreated skin to $13.28 \pm 0.38$ $\mu \mathrm{m}$ after application of the skin-care product. Moreover, Figure 3 suggests that the applied emulsion acts at the level of the outermost electron-lucent layer. Actually, Bar graph of Figure 3 proves that the mean thickness of that electron-lucent layer increases from $5.36 \pm 0.30 \mu \mathrm{m}$ for untreated skin to $8.34 \pm 0.27 \mu \mathrm{m}$ after application of the hand-cream emulsion. On Figure 5(b), one can note a layer of electron-lucent corneocytes covering underlying electron-dense cornified cells when the skin-care emulsion is applied after SLS exposure of the skin. Figure 1 points out that the mean thickness of the horny layer increases from $8.01 \pm 0.39 \mu \mathrm{m}$ for SLS treated skin (Figure 4(a)) to $13.55 \pm 0.81 \mu \mathrm{m}$ after topical application of the emollient (Figure 5(b)). That thickening process of the stratum corneum is particularly evident for the outermost electron-lucent layer (Figures 4(a), 5(b)). Figure 3 shows that the mean thickness of the electron-lucent layer increases from $0.62 \pm 0.19 \mu \mathrm{m}$ for SLS-exposed skin to $5.88 \pm 0.62 \mu \mathrm{m}$ after application of the skin-care

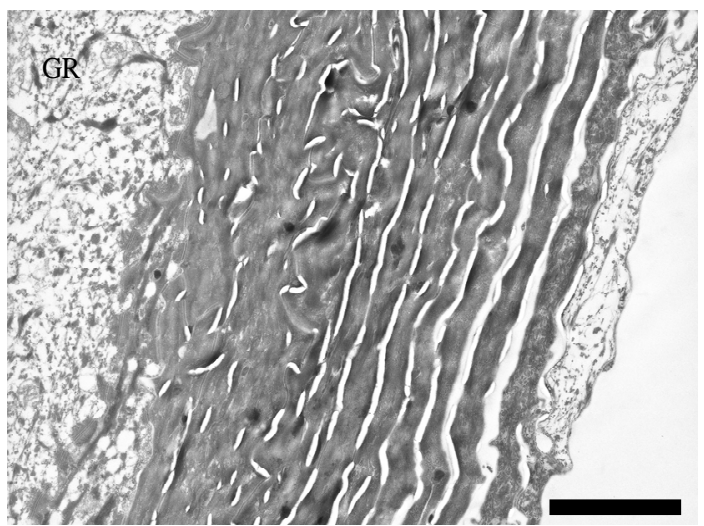

(a)

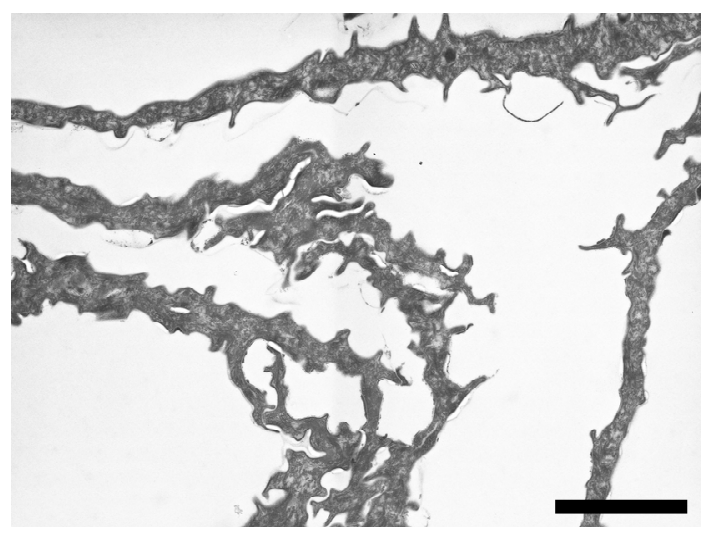

(b)

Figure 4. (a) TEM micrograph describing the structure of the stratum corneum after SLS treatment. Almost all superficial electron-lucent cells are shed from the skin surface. The outermost intercellular spaces of the electron-dense layer are widened. GR = granular layer. Scale bar represents $2 \mu \mathrm{m}$; (b) TEM micrograph describing the aspects of the desquamated corneocytes after SLS treatment. Individual or groups of electron-dense corneocytes detache from the skin surface. Cornified cell envelopes form spicules. Scale bar represents $2 \mu \mathrm{m}$.

emulsion. The thickening mechanism of the stratum corneum, induced by the topical application of the studied hand-cream, works with or without SLS irritation of the skin (Figure 1). It appears to result from a swelling phenomenon of the horny cells from both the outermost electron-lucent corneocytes and the innermost electron-dense cornified cells (Figures 6(a), 6(b)). The electro-lucent corneocyte thickness increases, after emollient application, from $0.69 \pm 0.04 \mu \mathrm{m}$ to $0.88 \pm 0.05 \mu \mathrm{m}$ without SLS treatment and from $0.49 \pm 0.04 \mu \mathrm{m}$ to 1.19 $\pm 0.08 \mu \mathrm{m}$ following SLS exposure (Figure 6(a)). Within the innermost electron-dense layer, the corneocyte thickness increases too, after hand-cream application, from $0.48 \pm 0.04 \mu \mathrm{m}$ to $0.64 \pm 0.04 \mu \mathrm{m}$ without SLS treatment and from $0.51 \pm 0.03 \mu \mathrm{m}$ to $0.99 \pm 0.05 \mu \mathrm{m}$ following 


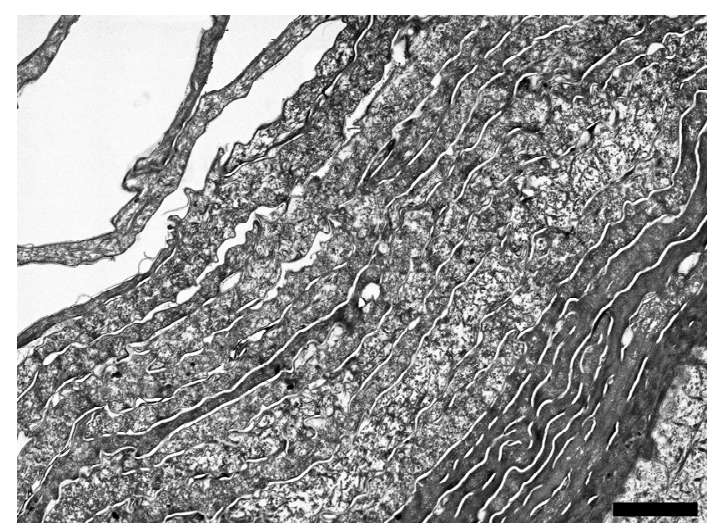

(a)

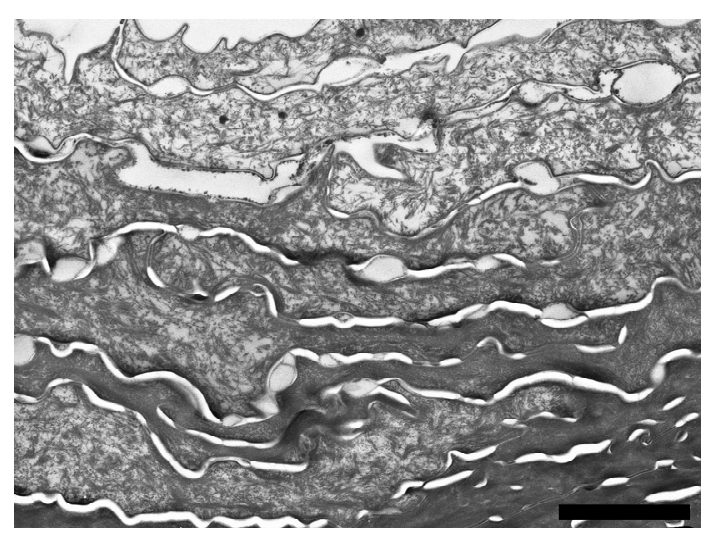

(b)

Figure 5. (a) Electron micrograph showing the structure of the stratum corneum after topical treatment with the emollient emulsion. Scale bar represents 2 um; (b) Electron micrograph showing the structure of the outermost part of the stratum corneum after SLS treatment followed by topical application of the emollient emulsion. Several electron lucent corneocytes cover the innermost electron-dense cornified layer. Scale bar represents $2 \mu \mathrm{m}$.

SLS exposure (Figure 6(b)). For both cell-layers (electron-lucent and electron-dense) the statistical differences are highly significant ( $\mathrm{p} \leq 0.001$ ) between the SLS exposed corneocytes and the subsequent emollient-treated cells. Statistical differences are also significant ( $\mathrm{p} \leq 0.05$ and $\mathrm{p} \leq 0.01$, respectively for the electron-lucent corneocytes and the electron-dense corneocytes) when the emollient-treated explants are compared to the control skin.

Figures 3 and 6(a) emphasize the deleterious effect of the irritant solution on the outermost cells. Statistical differences with the control skin are highly significant ( $p$ $\leq 0.01$ ). Moreover, it is noteworthy to stress that the restructuring effect of the stratum corneum, by topical application of the emollient emulsion, has the greatest impact on the outermost cell-layer of the epidermis (Figures 3, 6(a)).
Electron-lucent layer of stratum corneum

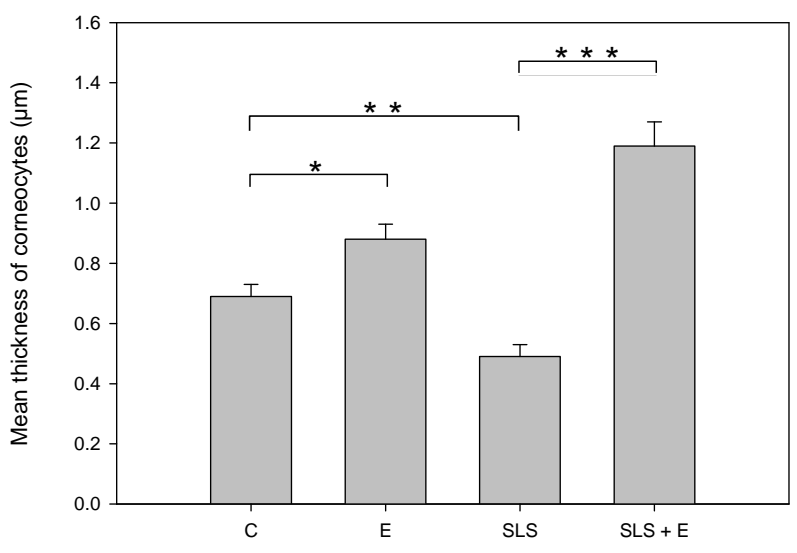

(a)

Electron-dense layer of stratum corneum

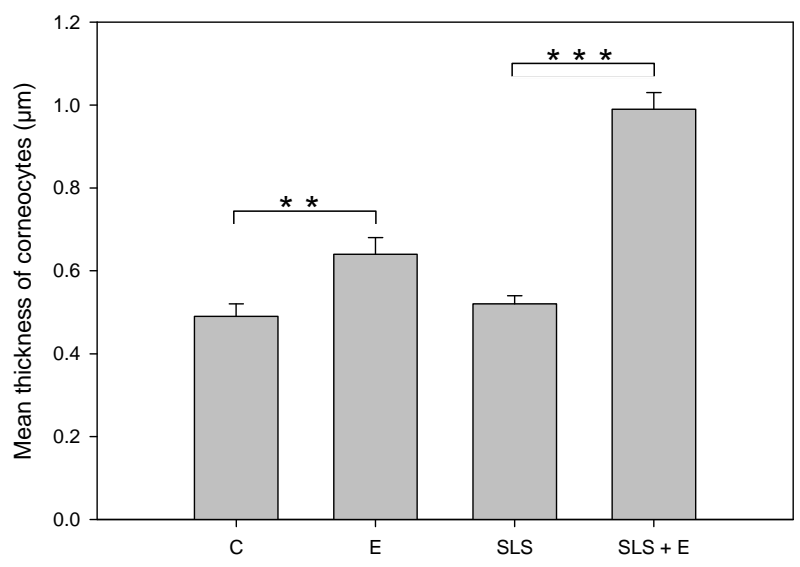

(b)

Figure 6. (a) Bar graph representing the mean thickness of the corneocytes of the electron-lucent layer of the stratum corneum vs skin treatment. Control specimen (C). $n=44$ measurements; emollient cream application (E), $n=59$ measurements; SLS treatment (SLS), $n=9$ measurements; emollient application after SLS exposure (SLS + E), $n=28$ measurements. Error bars represent the SEM; (b) Bar chart representing the mean thickness of the corneocytes of the electron-dense layer of the stratum corneum vs skin treatment. Control specimen (C). $n=484$ measurements; emollient cream application (E), $n=44$ measurements; SLS treatment (SLS), $n=94$ measurements; emollient application after SLS exposure (SLS + E), $n=55$ measurements. Error bars represent the SEM.

\section{Discussion}

Despite of recognized benefits on skin, we are still far from completely understanding the structural changes resulting from emollient applications. The goal of this study was to demonstrate that the effects of a hand-cream 
go beyond the cosmetic feel and appearance. With this in mind, we assessed, by TEM, the restructuring effects, at the cellular level, of the emollient emulsion after topical applications on normal and SLS-spoiled skin. The barrier function of the skin is obviously linked to the morphological architecture of the stratum corneum. In turn, a spoiled stratum corneum parallels a decline in barrier function. Here we investigated the mechanisms of SLS action on the stratum corneum integrity as well as the $e x$ vivo skin restructuring potential of the employed emulsion.

The bar graph of Figure 1 clearly indicates that the SLS exposure as well as the topical application of the hand-cream emulsion induced thickness changes of the stratum corneum. On one hand, Figure 1 indicates a thinning of the stratum corneum after SLS treatment; on the other hand the bar graph strongly suggests a thickening of the cornified layer when the emollient is applied on normal and SLS treated skin. White et al. (1987) likewise measured that the stratum corneum was thinned after washing the skin with soap [11]. SLS is an anionic surfactant known for long to have detrimental effects on all stratum corneum components [12]. It is a commonly used test substance for induction of skin barrier damage. Washes with concentrated solutions of SLS destroy the cutaneous barrier comprising the inter-corneocyte lipids [13]. The intercellular lipid lamellae can be seen when samples are post-fixed with ruthenium tetroxide $\left(\mathrm{RuO}_{4}\right)$ $[14,15]$. Several studies already addressed and documented the issue of extracellular space $[3,16,17]$. But here, we mainly focused the cellular architecture changes of the stratum corneum with respect to the different experimental conditions, i.e. normal skin, emollient-treated skin, SLS-exposed skin and SLS-exposed skin treated with the emollient emulsion.

Alteration of intracellular keratin matrix, cornified cell envelope and intercellular lipids may open both transcellular and intercellular routes across the stratum corneum [18]. Excessive exposure to surfactants damages the stratum corneum and can even lead to irritant reactions such as hand dermatitis [19].

As there is limited knowledge about the morphological effects induced by emollients, after topical application, we investigated in detail the features of the corneocytes of skin explants for different treatments. Thus we compared SLS-exposed skin and emollient treated tissue with non-treated explants, but we also followed the changes of SLS-exposed stratum corneum when treated with the hand-cream. First of all we noticed two different domains within the stratum corneum of the non-treated skin tissue. TEM observations clearly show an electron-lucent layer of corneocytes covering more electron-dense cornified cells (Figure 2(a)). Obviously the electron-dense ap- pearance of the innermost layer of the stratum corneum comes from the densely packed intracellular material of these cornified cells (Figures 2(a)-(c)). Apart the differences in electron-density of the corneocytes from these two domains, one can also note that so to say no more corneodesmosomes are present within the outermost electron-lucent layer of corneocytes, whereas corneodesmosomes are still observable between the cells of the underlying electron-dense layer (Figures 2(b), 2(c)). Quantitative analyses of the thicknesses of the outermost electron-lucent layers of the respective experiments (Figure 3) confirm the aforementioned results regarding SLS exposure and emollient treatment of the skin. The exposure of the skin explants to a $0.1 \%$ aqueous solution of SLS surfactant for $24 \mathrm{~h}$ severely reduces the thickness of the superficial layer of electron-lucent corneocytes. Desquamation of the stratum corneum has been associated with the progressive degradation of corneodesmosomes toward the surface [17]. Now, SLS-induced skin barrier defects induce altered mRNA expression of enzymes (kallikreins) involved in the desquamation process [20]. Kallikrein-7 and Kallikrein-5 have been shown to cleave corneodesmosal proteins [21]. The enzymatic degradation of corneodesmosomes weakens the intercellular cohesion of the stratum corneum and desquamation is likely to occur [17]. Our observations showing a pronounced release of corneocytes from the surface after SLS treatment (Figure 4(a)) are consistent with these previous research reports. The spicules of the desquamated corneocytes (Figure 4(a)) might also illustrate the alteration potential of the SLS solution at the cellular level.

On one hand surfactants, as SLS, are known to increase the fluidity of the skin barrier lipids [6], on the other hand oils (mineral, vegetable and/or animal) have been used since ancient times for the protection of the skin. Oil applications moisturize the skin by reinforcing the intercellular lipids of the stratum corneum and, in turn, providing a better barrier function. For instance, in vivo confocal Raman microspectroscopy evaluations have shown that paraffin oil (mineral oil) and vegetable oils can penetrate the top layers of the stratum corneum [22]. The latter measured from the water content profiles the thickness of the stratum corneum after topical applications of different oils. They measured an increase of the stratum corneum thickness of about $10 \%$. The present findings are consistent with these statements. Indeed, our results emphasize that the application of the emollient, containing $19 \%$ of fatty phase, thickens the stratum corneum, when applied on normal or SLS-treated skin explants (Figure 1). This effect is particularly evident at the level of the outermost layer of electron-lucent corneocytes (Figure 3). Although the architecture of the 
stratum corneum appears unchanged after topical use of the emollient (Figure 5(a)), it seems that the application of the emulsion, after SLS-exposure of the tissue, favours the cohesion of the superficial corneocytes, that in turn conducts to a thickening of that outermost cell layer (Figure 5(b)). On this matter, Lintner et al. (1997) showed that a synthetic ceramide was able to contribute to the restructuring of the stratum corneum of skin damaged by stripping or SLS washes [13].

To go further into the understanding of the restructureing mechanisms, we paid special attention to the cellular architecture of the stratum corneum for the different epidermal treatments. By measuring the thicknesses of the corneocytes from both layers (electron-lucent and electron-dense), we noticed that SLS exposure conducts to a significant thinning of the corneocytes essentially from the outermost electron-lucent layer of the stratum corneum. (Figures 6(a), 6(b)). The bristly aspect of the desquamated cells might result from this shrinkage process (Figure 4(b)). According to a previous report [23], after SLS exposure, the binding of surfactant molecules to stratum corneum proteins reduces the ability of the cornified tissue to bind and hold water. Interestingly, the use of the emollient leads to a thickening of the corneocytes of both the electron-lucent and the electron-dense layers of the stratum corneum (Figures 6(a), 6(b)). This swelling effect is particularly evident after SLS exposure (Figures 6(a), 6(b)). Moreover, it is worth mentioning that the action of the emollient concerns the whole stratum corneum (Figures 6(a), 6(b)).

In conclusion, this experimental model of ex vivo human skin allowed identifying the structural changes occurring in the stratum corneum of skin explants exposed to SLS and/or treated with the hand-cream. The present study completes the knowledge regarding the structural mechanisms underlying stratum corneum repair after chemically induced damage. Clearly, our results strongly suggest that both, SLS-induced damage and emollientdriven repair process take place at the cellular level of the stratum corneum. One can envisage that the structural changes observed after topical application of the handcream are likely to ameliorate or restore the barrier function of the stratum corneum. Furthermore the information regarding the restructuring effect of the emollient cream seems to indicate a potential way to promote barrier repair.

\section{REFERENCES}

[1] M. Turkoglu, E. Pekmezci and A. Sakr, "Evaluation of Irritation Potential of Surfactant Mixtures," International Journal of Cosmetic Science, Vol. 21, No. 6, 1999, pp. 371-382. doi:10.1046/j.1467-2494.1999.211920.x

[2] A. V. Rawlings, “Recent Advances in Skin 'Barrier' Re- search,” Journal of Pharmacy and Pharmacology, Vol. 62, No. 6, 2010, pp. 671-677.

[3] A. Al-Amoudi, J. Dubochet and L. Norlén, "Nanostructure of the Epidermal Extracellular Space as Observed by Cryo-Electron Microscopy of Vitreous Sections of Human Skin,” Journal of Investigative Dermatology, Vol. 124, No. 4, 2005, pp. 764-777. doi:10.1111/j.0022-202X.2005.23630.x

[4] M. Haftek, M.-H. Teillon and D. Schmitt, "stratum corneum, Corneodesmosomes and ex Vivo Percutaneous Penetration," Microscopy Research and Technique, Vol. 43, No. 3, 1998, pp. 242-249. doi:10.1002/(SICI)1097-0029(19981101)43:3<242::AIDJEMT6>3.0.CO;2-G

[5] A. M. Goldstein and W. Abramovits, "Ceramides and the stratum corneum: Structure, Function and New Methods to Promote Repair," International Journal of Dermatology, Vol. 42, No. 4, 2003, pp. 256-259. doi:10.1046/j.1365-4362.2003.01507.x

[6] C. R. Harding, A. Watkinson and A. V. Rawlings, "Dry Skin, Moisturization and Corneodesmolysis," International Journal of Cosmetic Science, Vol. 22, No. 1, 2000, pp. 21-52. doi:10.1046/j.1467-2494.2000.00001.x

[7] L. Couturier and F. Yvergnaux, "Combined Structural and Biological Activities for New Polyunsaturated Fatty Derivatives Obtained by Biotechnological Process," International Journal of Cosmetic Science, Vol. 31, No. 3, 2009, pp. 209-224. doi:10.1111/j.1468-2494.2009.00496.x

[8] M. Fartasch, "Epidermal Barrier in Disorders of the Skin,” Microscopy Research and Technique, Vol. 38, No. 4, 1997, pp. 361-372. doi:10.1002/(SICI)1097-0029(19970815)38:4<361::AIDJEMT4>3.3.CO;2-B

[9] M. Lodén and C. Wessman, "The Influence of a Cream Containing 20\% Glycerine and Its Vehicle on Skin Barrier Properties," International Journal of Cosmetic Science, Vol. 23, No. 2, 2001, pp. 115-119. doi:10.1046/j.1467-2494.2001.00060.x

[10] K. Levi, R. J. Weber, J. Q. Do and R. H. Dauskardt, "Drying Stress and Damage Processes in Human stratum corneum," International Journal of Cosmetic Science, Vol. 32, No. 4, 2010, pp. 276-293. doi:10.1111/j.1468-2494.2009.00557.x

[11] M. I. White, D. McEwan Jenkinson and D. H. Lloyd, "The Effect of Washing on the Thickness of the stratum corneum in Normal and Atopic Individuals," British Journal of Dermatology, Vol. 116, No. 4, 1987, pp. 525530. doi:10.1111/j.1365-2133.1987.tb05873.x

[12] A. W. Fulmer and G. J. Kramer, "stratum corneum Lipid Abnormalities in Surfactant-Induced Dry Scaly Skin," Journal of Investigative Dermatology, Vol. 86, No. 5, 1986, pp. 598-602. doi:10.1111/1523-1747.ep12355351

[13] K. Lintner, P. Mondon, F. Girard and C. Gibaud, "The Effect of a Synthetic Ceramide-2 on Transepidermal Water Loss after Stripping or Sodium Lauryl Sulphate Treatment: An in Vivo Study," International Journal of Cosmetic Science, Vol. 19, No. 1, 1997, pp. 15-25. 
doi:10.1111/j.1467-2494.1997.tb00162.x

[14] R. R. Warner, Y. L. Boissy, N. A. Lilly, J. S. Spears, K. McKillop, J. L. Marshall and K. J. Stone, "Water Disrupts stratum corneum Lipid Lamellae: Damage Is Similar to Surfactants," Journal of Investigative Dermatology, Vol. 113, No. 6, 1999, pp. 960-966. doi:10.1046/j.1523-1747.1999.00774.X

[15] S. J. Jiang, X. J. Zhou, G. Q. Sun and Y. Zhang, "Morphological Alterations of the stratum corneum Lipids Induced by Sodium Lauryl Sulphate Treatment in Hairless Mice,” Journal of Dermatological Science, Vol. 32, No. 3, 2003, pp. 243-246. doi:10.1016/S0923-1811(03)00134-8

[16] R. R. Warner, K. J. Stone and Y. L. Boissy, "Hydratation Disrupts Human stratum corneum Ultrastructure," Journal of Investigative Dermatology, Vol. 120, No. 2, 2003, pp. 275-284. doi:10.1046/j.1523-1747.2003.12046.x

[17] K. Levi, J. Baxter, H. Meldrum, M. Misra, E. Pashkovski and R. H. Dauskardt, "Effect of Corneodesmosome Degradation on the Intercellular Delamination of Human stratum corneum," Journal of Investigative Dermatology, Vol. 128, No. 9, 2008, pp. 2345-2347. doi:10.1038/jid.2008.107

[18] J.-N. Lee, S.-H. Jee, C.-C. Chan, W. Lo, C.-Y. Dong and S.-J. Lin, "The Effects of Depilatory Agents as Penetration Enhancers on Human stratum corneum Structures," Journal of Investigative Dermatology, Vol. 128, No. 9, 2008, pp. 2240-2247. doi:10.1038/jid.2008.82
[19] P. Dykes, “Surfactants and the Skin,” International Journal of Cosmetic Science, Vol. 20, No. 1, 1998, pp. 53-61. doi:10.1046/j.1467-2494.1998.171735.x

[20] H. Törmä, M. Lindberg and B. Berne, "Skin Barrier Disruption by Sodium Lauryl Sulfate-Exposure Alters the Expression of Involucrin, Transglutaminase 1, Profilaggrin and Kallikreins during the Repair Phase in Human Skin in Vivo," Journal of Investigative Dermatology, Vol. 128, No. 5, 2008, pp. 1212-1219. doi:10.1038/sj.jid.5701170

[21] J. P. Hachem, M. Q. Man, D. Crumrine, Y. Uchida, B. E. Brown, V. Rogiers, D. Roseeuw, K. R. Feingold and P. M. Elias, "Sustained Serine Proteases Activity by Prolonged Increase in $\mathrm{pH}$ Leads to Degradation of Lipid Processing Enzymes and Profound Alterations of Barrier Function and stratum corneum Integrity," Journal of Investigative Dermatology, Vol. 125, No. 3, 2005, pp. 510-520. doi:10.1111/j.0022-202X.2005.23838.X

[22] G. N. Stamatas, J. de Sterke, M. Hauser, O. von Stetten and A. van der Pol, "Lipid Uptake and Skin Occlusion Following Topical Application of Oils on Adult and Infant Skin,” Journal of Dermatological Science, Vol. 50, No. 2, 2008, pp. 135-142.

[23] K. P. Ananthapadmanabhan, D. J. Moore, K. Subramanyan, M. Misra and F. Meyer, "Cleansing without Compromise: The Impact of Cleansers on the Skin Barrier and the Technology of Mild Cleansing," Dermatologic Therapy, Vol. 17, Supplement 1, 2004, pp. 16-25. 